\title{
Background and Advantages of a Tiered EMS Response in a Large, Fire-Based EMS Model
}

\section{Katarzyna Kimmel ${ }^{1 *}$ and David Persse ${ }^{2}$}

${ }^{1}$ Department of Emergency Medicine, Baylor College of Medicine, EMS Fellow, Houston Fire Department, USA ${ }^{2}$ Medical Director, Houston Fire Department, Professor Emergency Medicine, Baylor College of Medicine, USA

\begin{abstract}
Emergency medical services (EMS) systems vary across country, state, and even county lines. Which model provides the most effective and safe care has been hotly debated. Some models employ a fire-based EMS response, whereas others are privately funded and are completely separate from other public responders. Response can be uniform or tiered, which means that either ALS units respond to every call or calls are triaged based on their severity and units are dispatched accordingly. This requires a sophisticated dispatch algorithm that screens for emergencies of highest priority. Persse et al. sought to determine the difference in survival in out-of-hospital ventricular fibrillation arrest between a tiered response EMS model and an all advanced life support (ALS) response model within the same system.
\end{abstract}

Emergency medical services (EMS) has evolved greatly since its inception. The model has gone from a load-and-go philosophy to one that integrates high-level medical knowledge and techniques. As the practice of prehospital medicine evolves, these models must also evolve to provide good care. This requires efficient utilization of resources and placement of these resources in areas that are quickly accessible to those in need. A proposed solution has been to dispatch units based on screening by 911 call-takers. EMS response can be grouped into two categories: uniform and tiered. Uniform response means an advanced life support (ALS) unit is always dispatched. A tiered response sends first responders, basic life support (BLS) units, and/or ALS units depending on how the caller answers a series of questions asked by the dispatcher. Persse et al. investigated the difference in survival of patients in the uniform versus tiered response areas within a single, large system. Survival rates for those with out-of-hospital ventricular fibrillation arrest, as well as time to skilled procedures were compared. They found that survival rates were higher in patients treated in the tiered response group. Time to skilled procedures was shorter as well. In this article we aim to provide a background for Persse et al's study and underscore its relevance [1].

\section{EMS Training}

Emergency medical technicians (EMTs) are trained at three main levels - basic, which fulfills first responder duties, intermediate, which incorporates some higher skills and knowledge, such as advanced airway techniques and intravenous line (i.v.) insertion, and paramedic, which includes advanced cardiac life support (ACLS) in addition to the aforementioned skills [2].

Training for paramedics is longer and requires a more advanced skill set that, in turn, requires more time and experience to master. ALS providers need repeated exposure to advanced interventions to maintain their skills [3]. Skill dilution has been an observed phenomenon in models with higher paramedic to population ratio, and some studies have shown a correlation between a smaller number of paramedics to BLS responders, and successful intubation rates [36]. Stout et al. similarly suggested that an all ALS response system causes skill dilution in paramedics, as most calls do not require ALS skills [7].

\section{Uniform vs Tiered Response}

The uniform response (one that includes a paramedic) provides some advantages, most notably that there is always an advanced practitioner available on scene. It is also easy to implement, and has been shown to be economically efficient [7-8]. A tiered response model calls for basic life support (BLS), ALS, and/or other EMS resources to be dispatched, depending on the information provided by the caller. Supporters of this model argue that paramedics are in fact not necessary for a majority of calls. Because this decreases paramedic usage, they are available to respond quickly to more complicated emergency situations. Evidence suggests that this model allows paramedics to keep their skill set current, as mentioned above [1-7].

\section{The Need for ALS}

Persse et al. view the ALS provider as an integral player in EMS. Researchers, however, have questioned the need for ALS in the prehospital setting, especially with the advent of the automated external defibrillator (AED) and decreased emphasis on medications in current ACLS guidelines [9]. One study in 1992 reported that less than twenty percent of 911 medical calls required paramedic level care [10]. A systematic review conducted in 2010 suggested that out of all presentations, epileptic patients and those in respiratory distress benefitted from ALS interventions, however the quality of the literature was poor [11]. Curka et al. demonstrated that out of BLS units dispatched in the observational period, only $1.6 \%$ of calls required ALS treatment prehospital [12]. BLS personnel generally over-report incidents and do not hesitate to call for back up, so the authors were confident that this was a conservative percentage. The most common procedures performed by paramedics were i.v. access and 12-lead monitoring in otherwise alert and stable patients, which suggests that these patients would have not had bad outcomes if these ALS interventions had not been performed [12]. Upon exclusion of the precautionary i.v., only $0.5 \%$ of BLS responses were true ALS upgrades. Advanced resuscitative therapy was only provided to 27

*Corresponding author: Katarzyna Kimmel, Assistant Professor, Emergency Medicine, Baylor College of Medicine, EMS Fellow, Houston Fire Department, USA, Tel: 713291 1134, E-mail: kasiakimmel@gmail.com

Received August 26, 2014; Accepted September 22, 2015; Published September 29, 2015

Citation: Kimmel K, Persse D (2015) Background and Advantages of a Tiered EMS Response in a Large, Fire-Based EMS Model. Health Care Current Reviews 3: 138. doi: 10.4172/2375-4273.1000138

Copyright: ( $2015 \mathrm{Kimmel} \mathrm{K,} \mathrm{et} \mathrm{al.} \mathrm{This} \mathrm{is} \mathrm{an} \mathrm{open-access} \mathrm{article} \mathrm{distributed} \mathrm{under}$ the terms of the Creative Commons Attribution License, which permits unrestricted use, distribution, and reproduction in any medium, provided the original author and source are credited. 
of 14,100 , or $0.2 \%$ of patients, in the tiered response. A study in Australia in a "two-tiered" (similar to Persse et al's tiered response) EMS system showed a benefit to patients who were treated by ACLS providers, but not necessarily by advanced interventions [13]. The authors stipulate that this could be due to increased medical knowledge on the part of the advanced providers. This suggests that ALS may be dispatched too frequently in the prehospital setting.

\section{ALS Interventions and Survival}

Few advanced prehospital interventions have been shown to have significant impact on survival. In some studies, prehospital administration of thrombolytics for myocardial infarction (MI) has been shown to improve survival [14]. Unfortunately, this is not a common practice in the United States. However overall rates of survival from out-of-hospital cardiac arrest have improved dramatically between 2005 and 2012 [9]. This is attributed to, in part, the modest increase in bystander CPR and use of AEDs in that time period. Chan et al. argue, though, that these factors do not appear to be the principal contributors. What was not studied was how the emphasis on better quality CPR affected survival, as well as guidelines underscoring the importance of uninterrupted CPR. The Cardiac Arrest Registry to Enhance Survival (CARES), from which this data are collected, does not take into account EMS response times or characteristics of EMS models. Similarly, researchers in the UK found no difference in survival and neurological outcomes between patients treated by BLS and ACLS responders in non traumatic out of hospital cardiac arrest in a twotiered system, but there was no comparison between a uniform and tiered model [15]. There are no studies that we could find investigating the impact on morbidity and mortality of the lack of availability of an advanced practitioner in the prehospital setting when one is indicated.

\section{Cardiac Arrest Survival as a Function of Ambulance Deployment Strategy}

Persse et al's paper sought to compare survival rates among witnessed ventricular fibrillation cardiac arrests in a large EMS system that provides both tiered and uniform response [1]. Other critical clinical performance indicators were recorded as well.

An observational study using prospectively collected data as part of a quality improvement program was performed [1]. The fire-based model uses a priority dispatch system that distinguishes between incidents that may require ALS and those that may not. The central part of the city utilizes 22 emergency medical technician (EMT) staffed BLS ambulances and 25 paramedic units, in addition to neighborhood EMTAED firefighter staffed fire apparatus (tiered response). The periphery of the response area is served by 7 paramedic units (ALS) with support from firefighter EMT-AED first responders (uniform response).

Reviewers calculated response and treatment intervention intervals from time of dispatch to arrival or intervention. Using the Utstein methodology, cardiac arrest survival data was collected prospectively [16]. Inclusion criteria were patients with witnessed collapse and intial rhythm of ventricular fibrillation. Patients with traumatic cardiac arrest, drug overdose, temperature extremes, obvious non-cardiac arrest, and age less than 18 were excluded. In the twelve-month study period, two hundred and five cases were included. Of these, one hundred eighty one victims of witnessed ventricular fibrillation cardiac arrest were in the targeted deployment area (tiered response), and the remainder in the uniform deployment region [1].

The analysis found that patients in the tiered response region had greater chance of survival to hospital discharge $-4.2 \%$ of patients survived in the uniform response group, and $23.9 \%$ in the tiered response ( $p$ value 0.03 ) [1]. This was also true for return of spontaneous circulation $(33.3 \%$ in uniform, $55.8 \%$ in tiered, $\mathrm{p}$ value 0.049$)$ and survival to hospital admission (29.2\% in uniform, $51.1 \%$ in tiered, $p$ value 0.05). Bystander CPR was more frequent in uniform response cases. Dispatch/arrival to shock interval was similar in both groups. Interestingly, dispatch and arrival to initiation of i.v. times were longer in the uniform group. The authors believe that this suggests decreased skill proficiency because time to intervention should be similar in cardiac arrest across response models. A similar phenomenon was also true for intubation - the uniform group's successful intubation was $91.9 \%$, and the tiered group made $99.4 \%$ of their intubations.

\section{Importance of Short Response Times}

Persse et al.s paper demonstrated that a tiered model lead to shorter response times for advanced personnel [1]. It seems intuitive that shorter response times will lead to better outcomes. However, this has not been uniformly shown to be true. Blackwell et al. studied priority 1 and 2 patients transported to a Level 1 trauma center, and found that there is no significant difference in median response times between survivors and non survivors [17]. There was a slight benefit of $1 \%$ when response times were less than five minutes. There was no evidence of increased mortality with an ALS response time over 10:59. The EMS model studied provided a uniform response. Important to note is that Blackwell et al.s study included patients with complaints from "back pain" to "unconscious" [17]. Conversely, Persse et al. examined survival in only those with ventricular fibrillation arrest [1]. In this subset of patients, early defibrillation has been shown to increase survival. The studied group in Blackwell et al.s paper could have provided a dilutional effect - whereas response times may not show benefit or harm in all comers, decreases in response times may be important for certain pathologies.

Advanced life support (ALS) response times have been demonstrated to be shorter when paramedic-staffed vehicles are available in addition to BLS responders [18]. One study in a Fire/EMS system with 3,261-call volume a month provided a quick response vehicle (QRV) staffed by a firefighter/paramedic staged in areas with high historical call volume percentages [17]. This EMS system provides uniform responses on a daily basis. The QRV was available during peak periods of $8 \mathrm{am}$ to $5 \mathrm{pm}$, and having it available, citywide average response time was reduced $27.62 \%$ (from 5.44 to 4.09 minutes).

\section{Tiered Models Implementation and Studies}

Targeted response provides efficient use of ALS resources [1012]. In order to implement a tiered model, dispatch personnel require algorithms to determine if a caller needs BLS or ALS [19]. Triage of EMS callers is a constantly evolving science. Dispatchers use combinations of medical history questions to identify the need for BLS or ALS response. Dispatch also determines whether adjunct personnel, such as rescue or hazardous material, are required.

A study done in King County, Washington in the 90's described the effects of conversion from chief complaint categories and subsequent response, to a Criteria Based Dispatch (CBD) [19]. Instead of dispatching units based on chief complaint, units were dispatched based on how they answered a series of questions, designed to identify those who would potentially require advanced treatment. For example, instead of a stroke patient classified in three finite categories - unconscious, having difficulty breathing or chest pain - callers were asked if the patient had any more specific complaints, such as if they are diabetic, had a seizure, 
severe headache, trouble speaking, and many other qualifiers. Based on answers, either ALS or BLS was dispatched. Culley et al. found a decrease in the number of ALS responses after implementation of CBD [19]. Similar codes were validated by Shah et al. who found that they accurately identified patients who required BLS [20].

Historically, Seattle and Milwaukee EMS were among the bestreported survival rates for cases of sudden death. Seattle operated with four medic units and Milwaukee with a similarly low number [12]. Researchers have attributed these results to the effects of a tiered EMS model on paramedic availability and skills. ALS units are more proficient at advanced skills because they spend more time practicing them on sick patients. For example, rates of successfully placed endotracheal tubes increased from $90 \%$ to $99.8 \%$ after conversion to a tiered model [12]. Response times were shorter for ALS units here, too. Investigators also found paramedic morale improved, attrition rates decreased, and there was a decrease in education expenses and cost of salaries [12].

\section{External Validity}

External validity of these findings is difficult to determine and will be exciting to investigate in other large, urban EMS services in the future. Unknown is the study's validity in regards to rural or remote areas. Presumably, given longer response times, a tiered system would be less effective and arguably dangerous. A tiered system requires an advanced dispatch system with frequent quality improvement measures, which may not be feasible in a rural setting [7]. Some researchers have suggested using location models - mathematical models that help maximize emergency services delivery to remote areas - as a possible solution to lack of timely EMS care in remote locations [21]. Integrating paramedics into the primary health care system, also known as community paramedicine, is another technique that has been described in addressing the lack of resources in the rural setting [22].

It is important to note that although there is some literature published regarding pros and cons of each model, varied characterization is another threat to external validity of the study. The terms "uniform" and "tiered" are not universally defined in the literature or in practice. Subsequently, some studies have investigated the benefits of a "tiered" model that does not bear the same definition as a tiered model in the paper discussed here. This makes drawing conclusions on a larger scale troublesome.

Also, for example, the EMS system studied by Curka et al. had a great amount of feedback loops incorporated into the system [12]. A fully integrated system in which EMS physicians staff ERs to which patients are delivered, as well as 24-hour-a-day hotlines for quality assurance and members of the quality assurance team working in delivering EDs created a constant feedback loop for EMS personnel [12]. Understandably, this type of feedback system is rare and probably impossible in large, urban models.

Lastly, a limitation of Persse et al.s study, and many studies like it, is the descriptive and observational quality of the study.

\section{Conclusion}

The tiered response in a large, fire department based EMS structure appears to improve survival in those with witnessed, out-of-hospital ventricular fibrillation arrest. Whether this is attributable to improved skill maintenance of paramedics in the system or to their increased availability is still in question. Other factors that could contribute to this increased survival are patient demographics and target hospital characteristics.

\section{References}

1. Persse DE, Key CB, Bradley RN, Miller CC, Dhingra A (2003) Cardiac arrest survival as a function of ambulance deployment strategy in a large urban emergency medical services system. Resuscitation 59: 97-104.

2. Tintinalli, J. E. Kelen G. D Stapczynski, J. S (2004). Emergency medicine A comprehensive study guide. New York McGraw-Hill, Medical Pub. Division.

3. Cobas MA, De la Peña MA, Manning R, Candiotti K, Varon AJ (2009) Prehospital intubations and mortality: a level 1 trauma center perspective. Anesth Analg 109: 489-493.

4. Ochs M, Davis D, Hoyt D, Bailey D, Marshall L, et al. (2002) Paramedicperformed rapid sequence intubation of patients with severe head injuries. Ann Emerg Med 40: 159-167.

5. Bulger EM, Copass MK, Sabath DR, Maier RV, Jurkovich JG (2005) The use of neuromuscular blocking agents to facilitate prehospital intubation does not impair outcomes after traumatic brain injury. J Trauma 58:718-724.

6. Katz SH, Falk JL (2001) Misplaced endotracheal tubes by paramedics in an urban emergency medical services system. Ann Emerg Med 37: 32-37.

7. Stout J, Pepe PE, Mosesso VN Jr (2000) All-advanced life support vs tieredresponse ambulance systems. Prehosp Emerg Care 4: 1-6.

8. Stout J, In Kueh AE (1994) Prehospital systems and medical oversight, 2nd (Edn). St Louis Mosby Lifeline 81-97.

9. Chan PS, McNally B2, Tang F2, Kellermann A2; CARES Surveillance Group (2014) Recent trends in survival from out-of-hospital cardiac arrest in the United States. Circulation 130: 1876-1882.

10. Stratton SJ (1992) Triage by emergency medical dispatchers. Prehosp Disast Med 7:263-267.

11. Ryynänen OP, lirola T, Reitala J, Pälve H, Malmivaara A (2010) Is advanced life support better than basic life support in prehospital care? A systematic review. Scand J Trauma Resusc Emerg Med 18: 62.

12. Curka PA, Pepe PE, Ginger VF, Sherrard RC, Ivy MV, et al. (1993) Emergency medical services priority dispatch. Ann Emerg Med 22: 1688-1695.

13. Woodall J, McCarthy M, Johnston T, Tippett V, Bonham R (2007) Impact of advanced cardiac life support-skilled paramedics on survival from out-ofhospital cardiac arrest in a statewide emergency medical service. Emerg Med J 24: 134-138.

14. McCaul M, Lourens A, Kredo T (2014) Pre-Hospital versus in-hospital thrombolysis for ST-elevation myocardial infarction. Cochrane Database Syst Rev 9:CD010191.

15. Joyce SM, Davidson LW, Manning KW, Wolsey B, Topham R Outcomes of sudden cardiac arrest treated with defibrillation by emergency medical technicians (emt-ds) or paramedics in a two-tiered urban ems system. Prehospital Emergency Care 2:13-17.

16. Cummins RO, Chamberlain DA, Abramson NS, (1991) Recommended guidelines from uniform reporting of data from out-of-hospital cardiac arrest The Utstein style. Annals of Emergency Medicine 20: 861-874.

17. Blackwell TH, Kline JA, Willis JJ, Hicks GM (2009) Lack of association between prehospital response times and patient outcomes. Prehosp Emerg Care 13: 444-450.

18. Anderson DW, Dhindsa HS1, Wan W2, Salot D3 (2015) Does the Implementation of an Advanced Life Support Quick Response Vehicle (QRV) in an Integrated Fire/EMS System Improve Patient Contact Response Time? Prehosp Disaster Med 30: 382-384.

19. Culley LL, Henwood DK, Clark JJ, Eisenberg MS, Horton C (1994) Increasing the efficiency of emergency medical services by using criteria based dispatch. Ann Emerg Med 24: 867-872.

20. Shah MN, Bishop P, Lerner EB, Davis EA (2005) Validation of EMS dispatch codes associated with low-acuity patients. Prehospital Emergency Care 8:106-108

21. Chanta S, Mayorga ME, McLay LA (2014) Improving emergency service in rura areas a bi-objective covering location model for EMS systems. Ann Oper Res 221:133-159.

22. Goodwin K, Tobler L (2013) Improving rural health state policy options. National Conference of State Legislatures. 